\title{
Mortality following exenteration for malignant tumours of the orbit
}

\author{
I Rahman, A Maino, A E Cook, B Leatherbarrow
}

Br J Ophthalmol 2005;89:1445-1448. doi: 10.1136/bjo.2005.072892

Background: Orbital exenteration is a rare, but disfiguring procedure reserved for the treatment of locally invasive malignancy or potentially life threatening orbital neoplasms, when less destructive techniques are inadequate. The authors report their experience and analyses of 64 cases of orbital exenteration performed over a 13 year period, looking specifically at key factors affecting mortality associated with such a destructive surgical procedure.

Methods: Records were reviewed retrospectively of all patients who had undergone exenteration of the orbit from 1 January 1991 to 1 April 2004 inclusive, at the Manchester Royal Eye Hospital. In all cases of deceased individuals, the cause of death was determined by liaison with the general practitioner and local health authority. Duplicate death certificates were requested for all deceased patients from the Registrar for Births, Deaths, and Marriages, Southport, UK. Kaplan-Meier analysis was used to estimate survival following exenteration.

Results: Overall, 1 year survival post-exenteration was high at $93 \%$. After 3 years this had fallen to $67 \%$, followed by $57 \%$ after 5 years, and $37 \%$ at 10 years. 13 patients died as a direct result of the orbital tumour. A further nine died of unrelated medical conditions, and two patients succumbed to malignant processes originating elsewhere in the body. There was no difference in survival rate at 3 years $(p=0.99)$ and 5 years $(p=0.454)$ between those with clear resection margins and those without.

Conclusion: In this study it was found that there was an overall mortality rate of $38 \%$ over 12 years. The presence of clear surgical margins, although reassuring for the surgeon, should not be regarded as an indication of cure. However, an overall 1 year survival of $93 \%$ and a 10 year survival of $37 \%$ are reassuring in that a proportion of individuals achieve surgical cure following exenteration. $38 \%$ of patients died as a result of other medical causes over the 12 year follow up.

O rbital exenteration is a rare, but disfiguring procedure reserved for the treatment of locally invasive or potentially lives threatening orbital neoplasms, when less destructive techniques are inadequate. Several large studies have described their experience with orbital exenteration. ${ }^{1-5}$ However, few have reported on long term survival. ${ }^{16}$

We report our experience and analyses of 64 cases of orbital exenteration performed over a 13 year period, looking specifically at key factors affecting mortality associated with orbital exenteration. We aim to quantify the role of clear surgical margins and tumour type on long term outcomes.

\section{METHOD}

Theatre records were reviewed retrospectively via a computerised database to identify all patients who had undergone exenteration of the orbit from 1 January 1991 to 1 April 2004 inclusive, at Manchester Royal Eye Hospital.

Patients surgical records were analysed and the following data recorded: age, sex, histological diagnosis, histological margins, the presence of recurrence or metastasis, and long term outcomes.

The cause of death was determined by liaison with the general practitioner and local health authority for those who had died. Duplicate death certificates were requested for all patients from the Registrar for Births, Deaths, and Marriages, Southport, UK.

Kaplan-Meier analysis was used to estimate survival following exenteration. Categorical variables were compared using contingency tables (Fisher's exact test). Prism statistical software was used throughout the study (GraphPad Software Inc, San Diego, CA, USA, Version 4.0).

\section{RESULTS}

A total of 64 orbits of 64 patients were identified from the database as having undergone orbital exenteration for malignant disease; 38 were females and 36 males. An equal distribution of 32 right and left eyes was noted. The age at exenteration ranged from 4-93 years (mean 70 years).

In the cohort of 64 patients, 14 different tumours were encountered. These are highlighted in table 1 .

The eyelids were the most common sites of tumour origin, accounting for 38 of the 64 exenterated patients. Malignant disease originating in the orbit and lacrimal gland (18), globe and conjunctiva (five), and three cases of unknown origin accounted for the remaining 26 cases.

Of the 64 orbits, 35 required a total orbital exenteration and a further a 21 subtotal exenteration (three with lid sparing procedures). In nine cases the procedure was not clearly documented. Morbidity/complications have been presented in a previous paper. $^{7}$

\section{Survival and long term follow up}

Follow up and subsequent course of the malignant disease to the time of writing was available in all 64 cases. Follow up to discharge ranged from 2-69 months (average: 19 months). Overall, 1 year survival post-exenteration was high, at $93 \%$. After 3 years this had fallen to $67 \%$, followed by $57 \%$ after 5 years, and $37 \%$ at 10 years (fig 1 ). Table 2 outlines the details of the 24 patients who died during the period of study. Thirteen patients died as a direct result of the orbital tumour. A further nine died of unrelated medical conditions, and two patients succumbed to malignant processes originating elsewhere in the body. A further two cases have metastatic

Abbreviations: BCC, basal cell carcinoma; SCC, squamous cell carcinoma 
Table 1 Histological diagnosis of patients undergoing exenteration

\begin{tabular}{ll}
\hline Histological diagnosis & $\begin{array}{l}\text { No of patients } \\
\text { (total = 64) }\end{array}$ \\
\hline Basal cell carcinoma & 28 \\
Sebacous cell carcinoma & 9 \\
Squamous cell carcinoma & 6 \\
Melanoma & 8 \\
$\quad$ Globe with extrascleral spread & 1 \\
Lids & 1 \\
Ethmoid sinuses & 2 \\
Adenocystic carcinoma (lacrimal gland) & 1 \\
Adenocarcinoma & 1 \\
Liposarcoma & 1 \\
Merkel cell & 1 \\
Lymphoma & 1 \\
Apocrine carcinoma & 1 \\
Rhabdomyosarcoma & 1 \\
Secondary metastasis, gastrointestinal primary & 1 \\
Malignant schwannoma & 1 \\
Malignant medulloepithelioma & \\
\hline
\end{tabular}

BCC, basal cell carcinoma; SCC, squamous cell carcinoma.

disease from ocular malignant melanomas at the time of writing.

Four patients had local recurrence. Histologically, two of these patients had basal cell carcinomas (BCCs), but did not achieve clear surgical margins. One case of secondary orbital metastasis displayed recurrence, again without clear surgical margins. The final patient had an adenocarcinoma of the lacrimal gland. Although clear surgical resection margins were achieved on histological examination, this patient underwent prophylactic orbital radiotherapy. A further 15 patients had regional or distant metastasis. Of these, there were seven cases of malignant melanoma, three sebacous cell carcinomas, two cases of SCC, one embryonal rhabdomyosarcoma, one Gorlin's syndrome, and one liposarcoma. All patients with melanoma had clear resection margins histologically. The remaining eight cases did not achieve clear resection margins, but all underwent adjuvant radiotherapy.

Overall, 34 patients achieved clear margins (group 1) compared to 30 who had residual tumour (group 2). Survival curves were not statistically significant $(\mathrm{p}=0.79)$ and are shown in figure 2 . The hazard ratio of 0.90 (95\% CI 0.40 to 2.01 ) shows that individuals in group 1 are dying at a rate of 0.90 compared to group 2. In group 1 , an overall 1 year survival rate of $97 \%$ was achieved. After 3 years this reduced to $67 \%$ and $53 \%$ after 5 years. This compared with $93 \%$ after 1 year, 67\% after 3 years, and 63\% after 5 years in group 2 . There is no difference in survival rate at 3 years $(p=0.99)$ and 5 years $(p=0.454)$ between the two groups.

Dividing the study group into those undergoing exenteration for BCCs, and those undergoing exenteration with other malignant processes, a similar pattern is observed as above (fig 3). The 1 year survival in the earlier group is $93 \%$, falling to $82 \%$ after 3 years, and $75 \%$ at 5 years. Comparatively, the latter group shows a survival of $97 \%$ after 1 year, declining to $58 \%$ after 3 years, and 52\% after 5 years. The difference in survival rates is again not significant $(\mathrm{p}=0.058$ after 3 years, $\mathrm{p}=0.077$ after 5 years, respectively). After 3 years, a BCC patient has a $30.4 \%$ likelihood of dying than a non-BCC patient. The relative risk increases to $37.2 \%$ after 5 years.

\section{DISCUSSION}

Exenteration of the orbit is both a radical and disfiguring procedure. For the surgeon, exenteration represents a final attempt at cure of a potentially lethal process. For the patient, the thought of sacrificing a prominent part of the face is neither comfortable nor comforting.

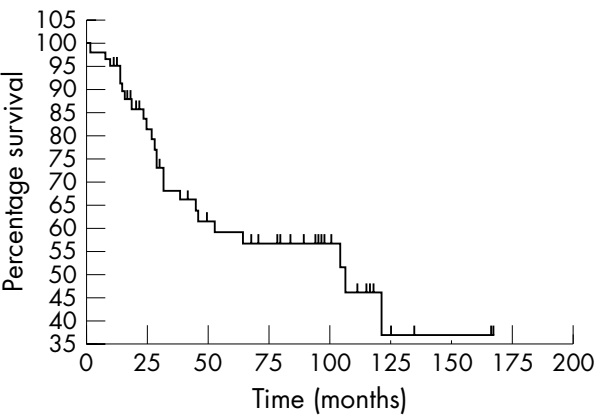

Figure 1 Survival rate in 64 cases of exenteration for orbital malignancy.

Exenteration as a curative procedure for many malignancies is debated. ${ }^{8-10}$ Few authors comment on the factors affecting survival. ${ }^{5611}$ For example, radical exenteration in cases of maxillary SCC with orbital invasion will naturally show a poor prognosis, as disease is already advanced and likely to have metastasised..$^{12}{ }^{13}$ However, in the absence of randomised controlled trials and large numbers of individual conditions, together with a natural bias towards exenteration of the more advanced cases, survival rates may not give a true picture and need cautious interpretation.

In our study, the overall survival after 5 years was $57 \%$. Others have reported a similar 5 year survival of $26-63 \% .^{5611}$ However, the case mix in all reported series represent significant differences and variability in histological type, patient characteristics, surgical procedure, and follow up. This said, these figures provide valuable statistics in patient counselling before surgery.

In our series, 28 patients had BCCs. 36 patients, therefore, had what may be regarded as more highly metastatic orbital neoplasms. However, Kaplan-Meier curves for these two groups revealed no significance in overall 5 year survival. Many of the deaths of patients in the BCC group (seven of eight cases) were unrelated to the BCC, and then succumbed to other medical conditions. Conversely, in the non-BCC group, malignant tumours of the orbit accounted directly for 12 of 16 fatalities. Death as a result of ageing does not appear to be a significant contributing factor in the two groups, as the average age of death in the BCC group was 68 (range 5091), compared to 65 (range 5-91). It can, therefore, be assumed that a similar prognosis maybe relevant in both groups at 5 years, regardless of the pathology.

Surgically clear margins are thought to be a key element in establishing surgical cure of a patient in any form of surgery for neoplasia. Our results confirm those reported by Mouriaux et al, ${ }^{6}$ in that tumour free margins have little effect on long term survival. This is somewhat surprising as the goal of tumour surgery relies largely on achieving clear margins. However, this apparent paradox confirms that many variables are involved in establishing cure. Clear excision margins may prevent local recurrence, but they do not prevent micrometastasis to lymph nodes or distant organs via the blood stream.

Our study reveals that five of the 10 cases $(50 \%)$ of malignant melanomas are dead as a result of metastatic disease. A further two have metastatic disease at present and three are free of tumour. However, nine cases had clear resection margins, suggesting early metastatic spread of the tumour. Pach et $_{\text {a }}{ }^{14}$ reviewed a series of 46 patients and found no benefit from exenteration compared to enucleation in long term survival. However, tumour size and ocular/orbital location were significant risk factors in survival. In a large study of 1607 cases, Starr and Zimmerman ${ }^{15}$ described a 5 year death rate of $66 \%$ in the presence of extrascleral 
Table 2 Cause of death in the 24 patients from death certificate entries

\begin{tabular}{|c|c|c|c|c|}
\hline Age/sex & $\begin{array}{l}\text { Time from exenteration } \\
\text { to death (months) }\end{array}$ & $\begin{array}{l}\text { Clear surgical } \\
\text { margins }\end{array}$ & Histological diagnosis & Cause of death \\
\hline $62 / M$ & 2 & $Y$ & $\mathrm{BCC}$ & Carcinoma of the lung \\
\hline $90 / F$ & 8 & $\mathrm{~N}$ & $\mathrm{BCC}$ & Myocardial infarction \\
\hline $5 / F$ & 10 & $\mathrm{~N}$ & Rhabdomyosarcoma & Rhabdomyosarcoma from orbit \\
\hline $82 / M$ & 14 & $\mathrm{~N}$ & Secondary metastasis & Gastrointestinal metastasis \\
\hline $67 / M$ & 14 & $\mathrm{~N}$ & Sebacous cell carcinoma & Metastasis from sebacous cell carcinoma \\
\hline $76 / M$ & 15 & Y & Melanoma & Metastasis from melanoma \\
\hline $51 / M$ & 16 & Y & $\mathrm{BCC}$ & Gorlin's syndrome \\
\hline $28 / F$ & 19 & $\mathrm{~N}$ & Liposarcoma & Metastatic liposarcoma from orbit \\
\hline $76 / F$ & 24 & Y & Melanoma & Metastasis from melanoma \\
\hline $83 / F$ & 25 & Y & SCC & Cardiac failure \\
\hline $87 / F$ & 27 & $\mathrm{~N}$ & $\mathrm{BCC}$ & Bronchopneumonia \\
\hline $83 / M$ & 28 & $\mathrm{~N}$ & SCC & Old age \\
\hline $52 / M$ & 29 & Y & Melanoma & Metastasis from melanoma \\
\hline $75 / F$ & 29 & $\mathrm{~N}$ & SCC & Metastatic carcinoma from eye \\
\hline $65 / M$ & 32 & Y & Lacrimal adenocarcinoma & Orbital adenocarcinoma \\
\hline $73 / M$ & 32 & $\mathrm{~N}$ & Sebacous cell carcinoma & Cerebral metastasis from sebacous cell carcinoma \\
\hline $87 / F$ & 39 & Y & Melanoma & Metastasis from melanoma \\
\hline $84 / M$ & 45 & Y & SCC & Bronchopneumonia \\
\hline $87 / F$ & 46 & Y & $\mathrm{BCC}$ & Ruptured myocardial infarction \\
\hline $73 / M$ & 53 & Y & Melanoma & Metastasis from melanoma \\
\hline $83 / F$ & 65 & Y & $\mathrm{BCC}$ & Chronic obstructive pulmonary disease \\
\hline $87 / F$ & 105 & $\mathrm{~N}$ & $\mathrm{BCC}$ & Carcinoma of colon \\
\hline $85 / M$ & 107 & $\mathrm{~N}$ & SCC & Motor neuron disease \\
\hline $80 / \mathrm{F}$ & 122 & Y & $\mathrm{BCC}$ & Myocardial infarction \\
\hline
\end{tabular}

extension. Rini et $a l^{10}$ raised the issue of exenteration in orbitally invasive melanoma having a potentially detrimental effect in survival. Confirming this, Affeldt $e t$ a $l^{16}$ and Kersten et $a l^{9}$ reported a poor long term survival even with early exenteration. As such, exenteration in the context of orbital melanoma has been superseded by more conservative measures. ${ }^{17}$

Similarly, the role of orbital exenteration in the treatment of adenocystic carcinoma of the lacrimal gland remains unclear. However, in the face of poor alternatives, options are often limited. Wright et a ${ }^{18}$ described 38 patients (of a series of 50) with malignant neoplasms of the lacrimal gland. Exenteration did not improve the survival or reduce the recurrence rate. However, with adjunctive radiotherapy and radical surgical resections survival tended to be longer. The poor prognosis of adenocystic carcinoma relates to early perineural spread. For cures to be effective, early spread of the tumour requires adjunctive therapy. Intra-arterial chemotherapy preoperatively has been postulated to be of benefit in this setting. ${ }^{19}{ }^{20}$ This said, in our series, two patients have undergone exenteration for adenocystic carcinoma. Both had clear resection margins histologically, and both individuals are still alive 10 years and 12 years postoperatively. Only one of the two underwent adjunctive radiotherapy.

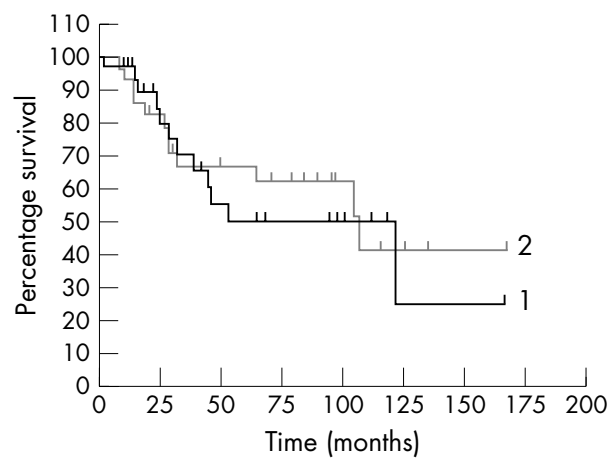

Figure 2 Survival curve comparing clear excision margins to unclear margins. ( 1 = clear excision margin group, $2=$ residual tumour group.)
The method of wound closure is an important cosmetic concern, but secondary to total, aggressive tumour resection. The argument for spontaneous granulation of the defect centres on the possibility of early detection of recurrence and donor site morbidity. However, our study does not support this theory, as local recurrence rates are small at four of 64 cases, supporting Mohr and Esser's ${ }^{1}$ observation of five of 74 cases with recurrence. Closure, therefore, may be planned preoperatively in discussion with patient concerns and likely prognosis of the condition.

\section{CONCLUSION}

Orbital exenteration should not be considered a curative procedure in most cases without adjunctive therapy. In cases of malignant melanoma and adenocystic carcinoma this should be thought of as palliative. However, the move towards more aggressive adjunctive therapy (chemotherapy and radiotherapy) may offer improved cure rates. Moreover, the presence of clear surgical margins, although reassuring for the surgeon, should not be regarded as an indication of cure.

In our study, we found a mortality rate of $38 \%$ over 12 years, of which $54 \%$ were associated with metastatic spread of the tumour. However, overall 1 year survival of $93 \%$

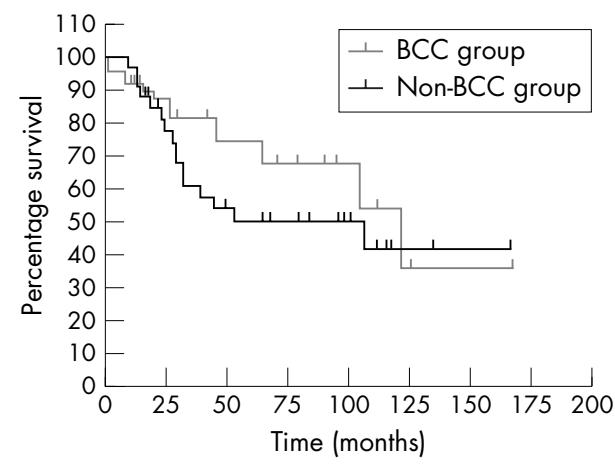

Figure 3 Survival curves comparing $B C C$ patients with non-BCC patients. 
and 10 year survival of $37 \%$ are reassuring as a proportion of individuals achieve cure following exenteration. Further, we confirm the favourable outcome associated with exenteration for BCC in this setting.

\section{Authors' affiliations}

I Rahman, A Maino, A E Cook, B Leatherbarrow, Manchester Royal Eye Hospital, Lister Centre, Nelson Street, Manchester M13 9WL, UK

Competing interests: none declared

Correspondence to: Imran Rahman, Manchester Royal Eye Hospital, Lister Centre, Nelson Street, Manchester M13 9WL, UK; imran1973@ tiscali.co.uk

Accepted for publication 6 June 2005

\section{REFERENCES}

1 Mohr C, Esser J. Orbital exenteration: surgical and reconstructive strategies. Graefes Arch Clin Exp Ophthalmol 1997;235:288-95.

2 Naquin HA. Exenteration of the orbit. Arch Ophthalmol 1954;51:850-62.

3 Rathbun JE, Beard C, Quickert MH. Evaluation of 48 cases of orbital exenteration. Am J Ophthalmol 1971;72:191-9.

4 Levin PS, Dutton JJ. A 20 year series of orbital exenteration. Am J Ophthalmol $1991 ; 112: 496-501$

5 Bartley GB, Garrity JA, Waller RR, et al. Orbital exenteration at the Mayo Clinic 1967-1986. Ophthalmology 1989;96:468-74.

6 Mouriaux F, Martinot V, Pellerin P, et al. Survival after malignant tumours of the orbit and periorbit treated by exenteration. Acta Ophthalmol Scand 1999:77:326-30.
7 Rahman I, Cook A E, Leatherbarrow B. Orbital exenteration: a 13 year Manchester experience. Br J Ophthalmol 2005;89:1335-40.

8 Polito E, Leccisotti A. Epithelial malignancies of the lacrimal gland: survival rates after extensive and conservative therapy. Ann Ophthalmol 1993;25:422-6.

9 Kersten RC, Tse DT, Anderson RL, et al. The role of orbital exenteration in choroidal melanoma with extrascleral extension. Ophthalmology 1985;92:436-43.

10 Rini FJ, Jacobiec FA, Hornblass A, et al. The treatment of advanced choroidal melanoma with massive orbital extension. Am J Ophthalmol 1987; 104:634-40.

11 Mohr C, Esser J. Orbital exenteration: surgical and reconstructive strategies. Graefes Arch Clin Exp Ophthalmol 1997;235:288-95.

12 Perry C, Levine PA, Williamson BR, et al. Preservation of the eye in paranasal sinus cancer surgery. Arch Otolaryngol Head Neck Surg 1988;1 14:632-4.

13 Stern SJ, Goepfert H, Clayman G, et al. Orbital preservation in maxillectomy. Otolaryngol Head Neck Surg 1993;109:111-15.

14 Pach JM, Robertson DM, Taney BS, et al. Prognostic factors in choroidal and ciliary body melanomas with extrascleral extension. Am J Ophthalmol 1986; 101:325-31

15 Starr HJ, Zimmermann LA. Extrasceral extension and orbital recurrence of malignant malanomas of the choroid and ciliary body. Int Ophthalmol Clin 1962;2:369-85.

16 Affeldt JC, Minkler DS, Azen SP. Prognosis in uveal melanoma with extrasceral extension. Arch Ophthalmol 1980;98:1975-9.

17 Blanco G. Diagnosis and treatment of orbital invasion in uveal melanoma. Can J Ophthalmol 2004:39:388-96.

18 Wright JE, Rose GE, Garner A. Primary malignant neoplasms of the lacrimal gland. Br J Ophthalmol 1992;76:401-7.

19 Meldrum ML, Tse DT, Benedetto P. Neoadjuvant intracarotid chemotherapy for treatment of advanced adenocystic carcinoma of the lacrimal gland. Arch Ophthalmol 1998;116:315-21.

20 Tse DT, Neff AG, Onofrey CB. Recent developments in the evaluation and treatment of lacrimal gland tumours. Ophthalmol Clin North Am 2000;13:663-81. 